\title{
Natalizumab May Improve Cognition and Mood in Multiple Sclerosis
}

\author{
C. Lang ${ }^{a} \quad$ C. Reiss ${ }^{a} \quad$ M. Mäurer ${ }^{a, b}$ \\ a Department of Neurology, University of Erlangen-Nuremberg, Erlangen, and ${ }^{\mathrm{b}} \mathrm{Caritas}$ Krankenhaus $\mathrm{GmbH}$, \\ Bad Mergentheim, Germany
}

\section{Key Words}

Multiple sclerosis • Cognition • Natalizumab · Course • Treatment

\begin{abstract}
Immunomodulating therapies may prevent relapses in multiple sclerosis and stabilize neurological status. However, little is known about the influence particularly of newer drugs on cognitive functions. We conducted an open-label prospective study to demonstrate whether natalizumab is apt to improve cognitive functions and mood in 29 patients tested psychometrically while under treatment for 6 months. We found improvements in some measures of attention, memory, mood, and well-being, but no deterioration, although patients suffered from their diseases for more than 10 years and had an EDSS score of 3.5. It is concluded that natalizumab is able to stabilize or improve cognition and mood even in longer-lasting multiple sclerosis.
\end{abstract}

Copyright $\odot 2012$ S. Karger AG, Basel

\section{Introduction}

Cognition is often impaired in multiple sclerosis (MS) and is a function of disease activity and progression in the early stages. Most patients suffer from attentional deficits at an early point [1], and at least $40 \%$ of patients display minor cognitive impairments at the time of first diagno- sis. During the course of the disease more than $65 \%$ develop subtle deficits $[2,3]$. Although gross cognitive decline is rare and dementia is the exception [4], cognition is of paramount importance for quality of life, social contacts, and professional abilities. Some studies have shown a beneficial effect of immunomodulating basic therapies on cognitive function including corticosteroids [5-7], claiming that this is due to a general reduction of inflammatory activity within the brain. Natalizumab is the most effective immunomodulating treatment of MS that is currently available. Almost $40 \%$ of patients treated this way display no further disease activity as shown by a retrospective analysis [8]. According to the concept that impairment of cognition is related to inflammatory disease activity, natalizumab treatment should affect this domain, too. We performed an open-label study to evaluate cognitive and related neuropsychological functions under this regimen.

\section{Patients and Methods}

Twenty-nine patients who were given an intravenous natalizumab treatment after several other therapies had failed or proved inefficient were seen in the special MS outpatient department of our hospital. Epidemiological data are shown in table 1.

All patients except 1 had received corticosteroids at one point or another during their disease, 22 interferon beta-1a, 15 glatiramer acetate, 9 interferon beta-1b, 9 mitoxantrone, 4 azathioprine, 1 intravenous immunoglobulin, 1 cytostatics, and 1 teriflunomide. While under natalizumab, none of the above-mentioned

\section{KARGER}

Fax +41613061234 E-Mail karger@karger.ch www.karger.com
(C) 2012 S. Karger AG, Basel 0014-3022/12/0673-0162\$38.00/0

Accessible online at: www.karger.com/ene apl. Prof. Dr. med. Dipl.-Psych. Christoph J.G. Lang

Department of Neurology, University of Erlangen-Nuremberg Schwabachanlage 6

DE-91054 Erlangen (Germany)

Tel.+49 91318534 339, E-Mail christoph.lang@uk-erlangen.de 
Fig. 1. Results of the nonverbal learning test (NVLT) and the auditory verbal learning test (AVLT) at three examination points. Given are raw scores (means \pm 95\% confidence intervals) reflecting the difference score (recognition correct positives minus false positives) for the NVLT and correct reproductions for the AVLT. The last results were normal.

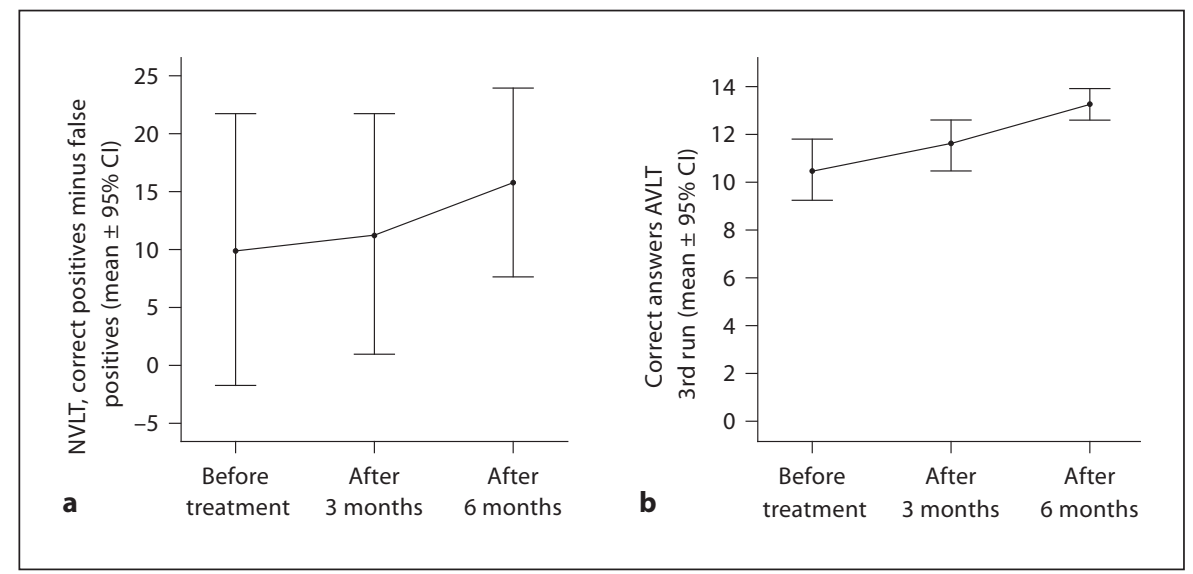

medication was given with the exception of 1 patient who suffered a relapse during the test interval and received high-dose intravenous cortisone temporarily. The natalizumab infusions were given at a constant dose $(300 \mathrm{mg})$ and at regular intervals (1 per month). One patient produced antibodies against natalizumab in the long run. Only 5 patients had to be given antibiotics and 2 patients analgesics for a limited time during the test period; the other concomitant medication remained unchanged (see table 1).

Testing was performed at 3-month intervals, i.e. before the first infusion (point 0), after 3 (point 1) and after 6 months (point 2).

Tests included the SF-36 scale (short-form health survey with 36 questions), a scale for physical and mental fatigue, a depression scale (Beck depression inventory, BDI-II [ed 2]), and cognitive tests such as the paced serial addition test (PASAT 3' form A), auditory verbal learning test (AVLT), divided attention (test battery for the assessment of attention, TAP), alertness (TAP), verbal learning test (VLT, Viennese test system), and non-verbal learning test (NVLT, Viennese test system). To keep conditions constant we used no parallel versions.

The main fields of our study were therefore memory, attention, and mood. Computer-based testing was used for maximum objectivity and reliability. Statistical evaluation was done using an ANOVA for dependent variables on a commercially available product (IBM SPSS statistics 19). Error probability was set at $\mathrm{p} \leq 0.05$.

\section{Results}

Many of the parameters tested showed significant improvements (NVLT, alertness, two SF-36 subscales [rolephysical, general health], BDI II, physical and mental fatigue, AVLT reproduction trials), the remainder was stable (divided attention, VLT, PASAT, other SF-36 subscales, AVLT recognition, phasic alertness). Altogether, 15 out of 30 parameters improved significantly, 15 remained equal, and none deteriorated. Examples are given in the figures.

Table 1. Characteristics of MS patients

$$
\text { Patients }(\mathrm{n}=29)
$$

Age, years

Sex

Schooling, years

Disease duration, years

MRI findings

Profession

EDSS

Concomitant medication in 10 patients
$33.7(19-54)$

24 female, 5 male

$11.0(9-21)$

$10.5(2-28)$

McDonald criteria

10 craftspeople, 19 service people $3.5(1.0-7.5)$

antihypertensives 1 , analgesics 2 , anti-spastics 2 , antibiotics 5 , antidepressants 2, antiepileptics 1 , hormones 1

Parentheses contain ranges, preceded by means. EDSS $=$ Expanded disability status scale.

A full display of results is given in table 2. Since not all tests could be given at all times to all patients, the number of available tests per time point varied between 29 and 20 . Using a Bonferroni correction for multiple measurements, the final level of significance was set at 0.0017. Significant results based on this criterion are given in bold face.

\section{Discussion}

MS results in motor, cognitive, and neuropsychiatric symptoms in a significant number of patients, all of which can occur independently of each other. Common cognitive symptoms include deficits in complex atten- 
Table 2. Overview of test results

\begin{tabular}{|c|c|c|c|c|}
\hline Time of test & Point 0 & Point 1 & Point 2 & ANOVA significance \\
\hline NVLT correct & $26.2 \pm 11.5$ & $30.8 \pm 5.0$ & $32.7 \pm 5.2$ & $0.016^{*}$ \\
\hline NVLT false & $18.2 \pm 11.1$ & $20.5 \pm 11.2$ & $16.9 \pm 9.1$ & 0.498 \\
\hline NVLT difference & $9.0 \pm 10.7$ & $10.3 \pm 9.7$ & $15.8 \pm 8.1$ & $0.047^{*}$ \\
\hline VLT correct & $33.0 \pm 8.0$ & $34.2 \pm 4.8$ & $34.9 \pm 3.4$ & 0.533 \\
\hline VLT false & $16.8 \pm 11.5$ & $19.7 \pm 10.7$ & $16.6 \pm 8.4$ & 0.500 \\
\hline VLT difference & $16.2 \pm 11.9$ & $14.5 \pm 11.3$ & $18.3 \pm 8.6$ & 0.506 \\
\hline Alertness without & $275.1 \pm 42.1$ & $245.5 \pm 28.0$ & $234.7 \pm 35.7$ & $0.000^{* *}$ \\
\hline Alertness with & $267.3 \pm 44.4$ & $232.6 \pm 26.9$ & $225.6 \pm 29.5$ & $0.000^{* *}$ \\
\hline BDI & $12.2 \pm 8.1$ & $7.7 \pm 8.1$ & $6.0 \pm 7.9$ & $0.022^{*}$ \\
\hline SF-36 PF & $55.4 \pm 28.3$ & $65.4 \pm 29.7$ & $70.0 \pm 28.1$ & 0.190 \\
\hline SF-36 RP & $35.7 \pm 43.8$ & $63.5 \pm 40.1$ & $75.0 \pm 37.9$ & $0.004^{* *}$ \\
\hline SF-36 BP & $78.1 \pm 27.0$ & $85.4 \pm 23.0$ & $85.1 \pm 27.8$ & 0.515 \\
\hline SF-36 GH & $39.9 \pm 16.5$ & $54.1 \pm 21.1$ & $53.7 \pm 24.2$ & $0.020^{*}$ \\
\hline SF-36 VT & $50.7 \pm 24.4$ & $56.3 \pm 18.3$ & $64.3 \pm 20.0$ & 0.093 \\
\hline SF-36 SF & $78.6 \pm 31.0$ & $82.7 \pm 26.7$ & $86.9 \pm 21.1$ & 0.566 \\
\hline SF-36 RE & $69.0 \pm 40.5$ & $82.0 \pm 38.0$ & $90.5 \pm 21.5$ & 0.106 \\
\hline SF-36 MH & $63.9 \pm 23.4$ & $68.0 \pm 18.6$ & $77.9 \pm 15.9$ & 0.052 \\
\hline SF-36 AH & $2.1 \pm .6$ & $3.2 \pm 0.9$ & $3.2 \pm 0.9$ & $0.000^{* *}$ \\
\hline SF-36 HC & $4.0 \pm 0.7$ & $2.3 \pm 1.0$ & $2.0 \pm 1.0$ & $0.000^{* *}$ \\
\hline SF-36 physical & $38.2 \pm 10.8$ & $44.5 \pm 10.8$ & $44.8 \pm 11.1$ & 0.053 \\
\hline SF-36 mental & $48.4 \pm 13.2$ & $49.9 \pm 10.4$ & $54.2 \pm 6.8$ & 0.167 \\
\hline Physical fatigue & $6.3 \pm 3.1$ & $1.0 \pm 1.7$ & $1.2 \pm 2.7$ & $0.000^{* *}$ \\
\hline Mental fatigue & $3.4 \pm 2.4$ & $1.2 \pm 1.4$ & $0.8 \pm 1.1$ & $0.000^{* *}$ \\
\hline AVLT1 & $7.2 \pm 2.0$ & $7.9 \pm 1.7$ & $9.0 \pm 1.6$ & $0.004^{* *}$ \\
\hline AVLT2 & $9.7 \pm 2.3$ & $10.7 \pm 2.2$ & $11.3 \pm 1.5$ & $0.017^{*}$ \\
\hline AVLT3 & $10.7 \pm 2.6$ & $11.9 \pm 2.4$ & $13.2 \pm 1.6$ & $0.001^{* *}$ \\
\hline AVLTR & $14.3 \pm 0.9$ & $14.5 \pm 0.9$ & $14.8 \pm 0.7$ & 0.253 \\
\hline Divided attention & $719.1 \pm 100.6$ & $713.6 \pm 94.3$ & $707.2 \pm 88.8$ & 0.909 \\
\hline PASAT & $40.6 \pm 10.2$ & $48.4 \pm 8.7$ & $48.9 \pm 13.9$ & $0.013^{*}$ \\
\hline
\end{tabular}

${ }^{*} \mathrm{p}<0.05,{ }^{* *} \mathrm{p}<0.01$. Results still significant after Bonferroni adjustment for multiple comparisons are given in bold face. Displayed are test points except for alertness and divided attention, where reaction times in milliseconds are given. Alertness without = Alertness without warning stimulus; Alertness with = alertness with warning stimulus; BDI = Beck depression inventory; AVLT = auditory verbal learning test; AVLT1 = first reproduction trial; AVLT2 $=$ second reproduction trial; AVLT3 $=$ third reproduction trial; AVLTR = recognition trial; NVLT = nonverbal learning test; PASAT = paced auditory serial addition test; VLT = verbal learning test; SF-36 = short-form health survey containing 36 questions $(\mathrm{PF}=$ physical functioning; $\mathrm{RP}=$ role-physical; $\mathrm{BP}=$ bodily pain; $\mathrm{GH}$ = general health; $\mathrm{VT}$ = vitality; $\mathrm{SF}=$ social functioning; $\mathrm{RE}=$ role-emotional; $\mathrm{MH}=\mathrm{mental}$ health; $\mathrm{AH}=$ actual heath; $\mathrm{HC}=$ health change; SF-36 physical = physical component summary; SF-36 mental = mental component summary).

tion, efficiency of information processing, executive functioning, processing speed, and long-term memory [9]. Other neurobehavioral and neuropsychological functions such as mood or mental well-being are also involved.

Studies on the effect of basic immunomodulatory treatment are very heterogeneous. In one study, a significant beneficial effect of interferon beta-1a was shown for processing speed, learning, and memory; interferon beta-
$1 \mathrm{~b}$ has shown some mild benefit, whereas glatiramer acetate showed no significant effect so far. It is believed that the effect of immunomodulatory drugs on cognition is mediated by their impact on brain inflammation. This may be the reason for the observation that it needs some time to develop a beneficial effect.

In our study, we observed the development of cognitive and neurobehavioral functions of patients treated with natalizumab, which is considered a very effective 
Fig. 2. Alertness with and without warning signal (median time in milliseconds) at three examination points. Given are medians $\pm 95 \%$ confidence intervals. The last results were near normal.

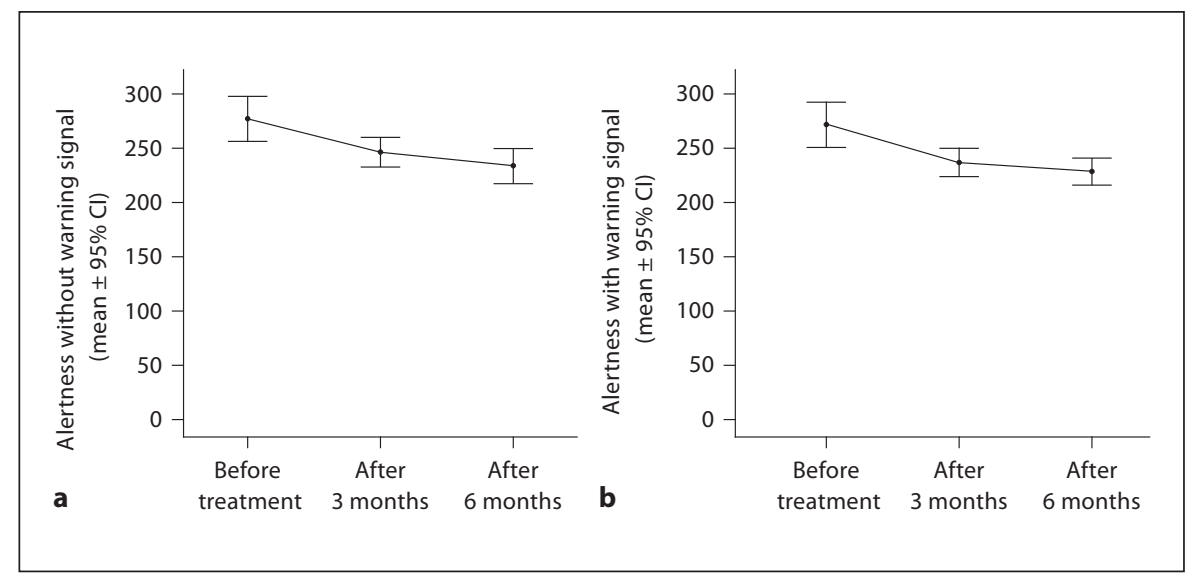

and fast-acting immunomodulatory agent. We could show that an effect on cognition was already present after 6 months of treatment.

Our results are in line with a recent study using brief cognitive tests to monitor impairments in patients with MS such as show up on a symbol digit modalities test (SDMT) and a MS neuropsychological screening questionnaire (MSNQ). The application of these tests has demonstrated the reliability and feasibility of short psychometric assessments as a monitoring tool for cognition over time [10]. Following a cohort of 713 patients over 48 weeks, mild but steady improvements were shown in the SDMT while the MSNQ - indicating cognitive problems - decreased at the same time.

This tendency was confirmed by our study. Of six cognitive tests applied, four improved, while two remained stable and none deteriorated. Of three questionnaires assessing subjective health, well-being and quality of life, two improved and one remained stable displaying a tendency for improvement. At the same time, the neurological outcome was favorable, since with one exception nobody experienced a relapse during the observation period. It has been demonstrated in turn that an elective interruption of natalizumab treatment after 24 months for 2-6 months resulted in a clinical relapse rate of $19 \%$ and an increased MRI activity in $47.4 \%$ [11]. Therefore, a sustained and regular therapy is advisable for an extended clinical effect and prevention of relapse.

Our findings are in agreement with a recent preliminary study in 17 patients presenting with a mean EDSS of 5 using a different test battery [12] and spanning a period of 1 year. Here, too, improvements were shown in memory and executive functions.
Although learning effects cannot be excluded in our study since no parallel test versions and no matched control group were available, the generally favorable outcome is strongly indicative of a positive influence on attention, memory, mood and subjective well-being. An ANOVA for dependent samples yielded significant differences comparing the first and last assessments for non-verbal learning, verbal reproduction, alertness, depression, fatigue, and working memory. Phasic alertness, i.e. the extent to which a warning signal adds to attentional performance, remained stable. The fields where no significant improvements were found were verbal recognition memory, divided attention, and most parameters of functional health.

These results emerged although the patients had formerly been treated with various other drugs without satisfying results, suffered from their disease for a mean of more than 10 years and showed a moderate disease severity (EDSS 3.5).

Summing up, natalizumab seems to be a promising treatment option for MS patients not only with regard to relapse prevention and neurological impairment, but also for cognitive and emotional functions.

Since this was an open-label prospective study, further work is necessary to prove whether this regime is superior to no or intermittent treatment or to treatment with other disease-modifying or immunomodulatory regimens. 


\section{References}

1 Tinnefeld M, Wilhelm H, Daum I, Faustmann PM, Haase CG: Cognitive deficits in early relapsing-remitting multiple sclerosis [In German]. Akt Neurol 2008;35:2-7.

2 Freedman MS: Long-term follow-up of clinical trials of multiple sclerosis therapies. Neurology 2011;76(suppl 1):S26-S34.

3 Tiemann L, Penner IK, Haupts M, Schlegel U, Calabrese P: Cognitive decline in multiple sclerosis: impact of topographic lesion distribution on differential cognitive deficit patterns. Mult Scler 2009;15:1164-1174.

4 Calabrese P, Penner IK: Cognitive dysfunctions in multiple sclerosis - a 'multiple disconnection syndrome?' J Neurol 2007; 254(suppl 2):II18-II21.

5 Barak Y, Achiron A: Effect of interferon-beta-1b on cognitive functions in multiple sclerosis. Eur Neurol 2002;47:11-14.
6 Patti F, Amato MP, Bastianello S, Caniatti L, Di Monte E, Ferrazza P, Goretti B, Gallo P, Morra VB, Lo Fermo S, Picconi O, Tola MR, Trojano M; COGIMUS Study Group: COGIMUS Study Group. Effects of immunomodulatory treatment with subcutaneous interferon beta-la on cognitive decline in mildly disabled patients with relapsing-remitting multiple sclerosis. Mult Scler 2010;16:68-77.

7 Patti F, Leone C, D’Amico E: Treatment options of cognitive impairment in multiple sclerosis. Neurol Sci 2010;31(suppl 2):S265S269.

8 Havrdova E, Galetta S, Hutchinson M, Stefoski D, Bates D, Polman CH, O'Connor PW, Giovannoni G, Phillips JT, Lublin FD, Pace A, Kim R, Hyde R: Effect of natalizumab on clinical and radiological disease activity in multiple sclerosis: a retrospective analysis of the Natalizumab Safety and Efficacy in Relapsing-Remitting Multiple Sclerosis (AFFIRM) study. Lancet Neurol 2009;8:254260.
9 Chiaravalloti ND, DeLuca J: Cognitive impairment in multiple sclerosis. Lancet Neurol 2008; 7:1139-1151.

10 Borriello G, Prosperini L, Marinelli F, Fubelli F, Pozzilli C: Observations during an elective interruption of natalizumab treatment: a post-marketing study. Mult Scler J 2010;17: 372-375.

11 Morrow SA, O’Connor PW, Polman $\mathrm{CH}$, Goodman AD, Kappos L, Lublin FD, Rudick RA, Jurgensen S, Paes D, Forrestal F, Benedict RH: Evaluation of the symbol digit modalities test (SDMT) and MS neuropsychological screening questionnaire (MSNQ) in natalizumab-treated MS patients over 48 weeks. Mult Scler 2010;16:1385-1392.

12 Mattioli F, Stampatori C, Capra R: The effect of natalizumab on cognitive function in patients with relapsing-remitting multiple sclerosis: preliminary results of a 1-year followup study. Neurol Sci 2011;32:83-88. 